\title{
A peculiar case of thrombotic thrombocytopenic purpura associated with falciparum malaria and successfully treated with plasma exchange
}

\author{
Authors: Ratnadeep Ghadge ${ }^{A}$ and Ajay Khobragade ${ }^{B}$
}

\section{Case presentation}

A 50-year-old woman was admitted to hospital 3 weeks prior with acute febrile illness. She was diagnosed and treated for falciparum malaria and then discharged. Following discharge, the patient developed altered sensorium, oliguria and seizures. She was admitted to another hospital where she was diagnosed with sepsis and uraemic encephalopathy. She was treated with intravenous antibiotics and dialysed, then discharged. She was readmitted to the same hospital on the following day with seizures and agitation. She was started on anti-epileptics. As there was no improvement in her mental status after 10 days, the patient was transferred to our hospital. The patient was observed to have high-grade fever, agitated behaviour, ongoing seizures and oliguria. She was investigated and found to have anaemia; raised creatinine and urine analysis showed plenty of red blood cells. No malarial parasite was detected in the peripheral smear. Pan-cultures were negative. Cerebrospinal fluid analysis was normal. Imaging studies like chest X-ray, ultrasonography of the kidney, ureter and bladder and magnetic resonance imaging of the brain were normal.

\section{Discussion}

The patient was reviewed with all investigations and found to have a pentad of fever, anaemia, thrombocytopenia, kidney injury and neurological involvement, raising suspicion about thrombotic thrombocytopenic purpura (TTP). To confirm the diagnosis, the patient was further evaluated. Lactate dehydrogenase was raised significantly and haptoglobin was reduced, suggestive of intravascular haemolysis. Peripheral smear showed occasional schistocytes, indicating microangiopathy. This confirmed the diagnosis of TTP.

\section{Conclusion}

The patient was started on plasmapheresis and steroids. In total, five plasma exchanges were given. After the third plasma exchange, neurological improvement was seen. Haematological improvement was seen after the fifth plasma exchange. The patient responded favourably to the treatment. The patient was discharged on oral steroids after a full recovery.

\section{Conflicts of interest}

None declared. 